\title{
MONITORANDO O DESEMPENHO AMBIENTAL DAS ORGANIZAÇÕES ATRAVÉS DA PRODUÇÃO MAIS LIMPA OU PELA AVALIAÇÃO DO CICLO DE VIDA
}

\author{
MONITORING THE ENVIRONMENTAL ACTING OF THE \\ ORGANIZATIONS THROUGH THE CLEAN PRODUCTION OR FOR \\ THE LIFE CYCLE ASSESSMENT
}

\author{
Roberta Tomasi Pires Hinz \\ Coordenadora e Professora do Curso de Tecnologia em Qualidade e Produtividade Industrial \\ - SOCIESC, Mestre em Ciência e Engenharia de Materiais \\ UDESC - Universidade do Estado de Santa Catarina \\ Departamento de Engenharia Mecânica \\ R. Alvino Wodtke, 99999, Bom Retiro - Joinville - SC \\ (47) 9961-1456 - roberta_pires@sociesc.com.br
}

\section{Luiz V. Dalla Valentina}

Coordenador e Professor do Curso de Engenharia Mecânica e Professor do Mestrado em

Ciência e Engenharia de Materiais

UDESC - Universidade do Estado de Santa Catarina

Departamento de Engenharia Mecânica

R. Alvino Wodtke, 99999, Bom Retiro - Joinville - SC

(47) 9974-9476 - dem2ldv@joinville.udesc.br

\section{Ana Claudia Franco}

Estudante de Engenharia de Produção de Sistemas

UDESC - Universidade do Estado de Santa Catarina

Departamento de Engenharia Mecânica

R. Alvino Wodtke, 99999, Bom Retiro - Joinville - SC

(47) 8806-6014 - analolaaa@ hotmail.com

Resumo: A sustentabilidade do planeta trata-se de uma responsabilidade coletiva e ações para melhorar o ambiente global são necessárias, isto inclui a adoção de práticas de produção e consumo sustentáveis. $\mathrm{O}$ objetivo deste artigo é realizar uma análise crítica entre a Produção mais Limpa (PmaisL) e a Avaliação do Ciclo de Vida (ACV), que são ferramentas imprescindíveis para o desenvolvimento sustentável do meio ambiente, incluindo aspectos sociais, econômicos e ambientais. Além de relatar a importância do envolvimento das partes interessadas (governo, indústria e sociedade), propõe-se alertar para a responsabilidade social atrelada a individualidade de cada um, que está em crescente interesse mundial, através de pesquisa teórica e estudos de caso. Informações comparativas entre a Produção mais Limpa e 
a Avaliação do Ciclo de Vida foram levantadas para apresentar afinidades ou não entre estas ferramentas. Constatou-se que a PmaisL está mais focada com a redução de resíduos gerados internamente, enquanto a ACV engloba todo o ciclo de produção, desde a extração da matéria-prima até a disposição do produto e seu retorno ao meio ambiente.

Palavras-chave: Avaliação do Ciclo de Vida, Produção mais Limpa, Preservação ambiental, Desenvolvimento sustentável.

Abstract: The sustainability of the planet is treated as a collective responsibility and actions are necessary. These actions include the adoption of sustainable practices of production and consumption. The objective of this paper is to make a critical analysis of the Clean Production $(\mathrm{P}+\mathrm{L})$ and the Life Cycle Assessment (LCA), those are indispensable tools for the sustainable environmental development including social, economical and environmental aspects. Besides telling the importance of the involvement of concerned groups (government, industries and society), we also alert to the social responsibility attached to each individual. There has been an increasing interest in this issue all over the world through theoretical research and case studies. A comparison between the Clean Production and the Life Cycle Assessment has been made so as to find out whether there are similarities between them (or not). It was concluded that the $\mathrm{P}+\mathrm{L}$ is more focused on the reduction of internally generated residues while the LCA includes the whole production cycle, from the extraction of raw material to the disposition of the product and its return to the environment.

Key-words: Life Cycle Assessment, Clean Production, Environmental Preservation, Sustainable Development.

\section{INTRODUÇÃO}

Diante do grande consumo de recursos naturais, o que acarreta uma previsível escassez de água potável, a perda da biodiversidade e o aumento da poluição, sobretudo no que diz respeito à mudança climática global, um número cada vez maior de empresas vem incorporando em suas estratégias o conceito da sustentabilidade. As empresas são alvo de novas expectativas quanto as suas responsabilidades para com a sociedade como agentes que dispõem de recursos financeiros, organizacionais e tecnológicos para uma atuação mais ágil, decisiva e direta na solução dos problemas ambientais e sociais. Consciente desta situação, metodologias fundamentais para implementar mudanças e práticas de desenvolvimento sustentável foram desenvolvidas. Entre elas, para este artigo, foram analisadas a Produção mais Limpa e a Avaliação do Ciclo de Vida.

O planeta está passando por mudanças drásticas como conseqüência do desenvolvimento da humanidade. Dessa forma, as empresas e a sociedade precisam se preocupar com a extração dos recursos naturais não-renováveis, através de um gerenciamento adequado, que considere também os ecossistemas envolvidos e inclua sua capacidade de 
recuperação, conciliando uma relação benéfica junto ao meio-ambiente. Portanto, a questão chave para as empresas, é: Como definir e aplicar estratégias para garantir seu desenvolvimento sustentável? Nesta percepção, em que os problemas ambientais estão associados à produção industrial, torna-se necessário trabalhar e aplicar conceitos de ecoeficiência.

Apesar de amplo e até mesmo complexo, é possível realizar uma análise para o desenvolvimento sustentável, através de ferramentas que auxiliam na coleta de dados e interpretação dos resultados. Entre elas, a Avaliação do Ciclo de Vida (ACV) pode ser considerada uma das mais apropriadas, pois busca soluções para problemas ambientais globais, uma vez que inclui todas as etapas do ciclo de vida de um produto ou processo, desde: a extração e processamento de matérias-primas, fabricação e embalagem, transporte e distribuição, uso e reemprego, reciclagem ou reutilização até a disposição final. Outra ferramenta muito utilizada nas empresas é a Produção mais Limpa, também conhecida como PmaisL, que trata de análises e ações ambientais preventivas, economizando água, energia e matérias-primas. Este artigo, propõe uma análise crítica entre a Produção mais Limpa e a Avaliação do Ciclo de Vida, para auxiliar as organizações na tomada de decisões ambientalmente corretas.

\subsection{Avaliação do Ciclo de Vida}

A ACV surgiu da necessidade de se estabelecer uma metodologia que facilitasse a análise e os impactos ambientais entre as atividades de uma empresa, incluindo seus produtos e processos. A partir dessa metodologia, pode-se verificar que a prevenção à poluição se torna mais racional, econômica e efetiva do que uma ação na direção dos efeitos gerados. Um dos objetivos da ACV é estabelecer uma sistemática confiável e que possa ser reproduzida a fim de possibilitar a decisão entre várias atividades, aquela que terá menor impacto ambiental.

Pela SETAC (Society of Envirommental Toxicology and Chemistry), a avaliação do ciclo de vida é um processo objetivo de avaliar as cargas ambientais associadas com um produto, processo ou atividade através da identificação, quantificação e avaliação de impactos quanto ao uso de energia e matéria e de emissões ambientais, e a determinação de oportunidades de melhorias ambientais. A avaliação inclui todo o ciclo de vida do produto, processo ou atividade, envolvendo extração e processamento de matérias-primas; fabricação, 
transporte, e distribuição; uso/reuso/manutenção; reciclagem; e disposição final (DUARTE, 1997).

A busca constante pelo desenvolvimento sustentável é um dos maiores desafios para sobrevivência da humanidade. Em decorrência disso, considerando no âmbito internacional, surgiu a necessidade de estabelecer alguns padrões normativos para regulamentar os aspectos de controle ambiental. A norma NBR ISO 14040 (2006), padronizou e estabeleceu internacionalmente a definição para Avaliação do Ciclo de Vida, como sendo: a compilação e avaliação das entradas, das saídas e dos impactos ambientais potenciais de um sistema de produto ao longo de seu ciclo de vida, desde a aquisição da matéria-prima ou geração de recursos naturais à disposição final.

Segundo a Norma ISO 14040, o estudo da ACV está divido em quatro etapas:

a) definição do objetivo e escopo;

b) análise do inventário;

c) avaliação de impacto; e

d) interpretação dos dados e resultados.

Essas etapas foram estabelecidas a fim de se obter uma visão geral do processo. Chehebe (1998) faz a inter-relação entre estas fases através da Figura 1.

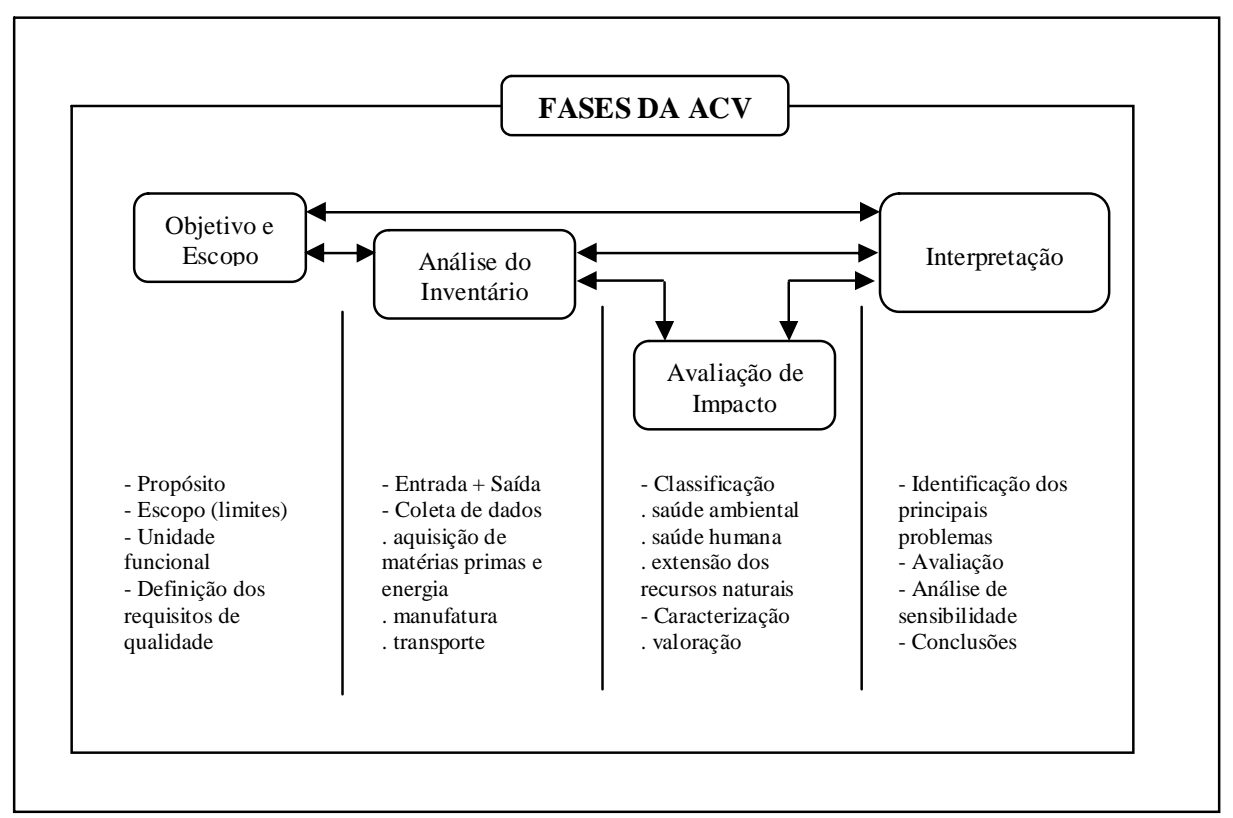

Figura 1: Fases da ACV Fonte: Chehebe, 1998. 
Dessa forma, a ACV, supera a visão focada apenas ao processo produtivo, no tratamento e disposição dos dejetos gerados durante a fabricação, para uma visão holística de todos os impactos ambientais associados a todas as fases do ciclo de vida de um produto (ver Figura 2).

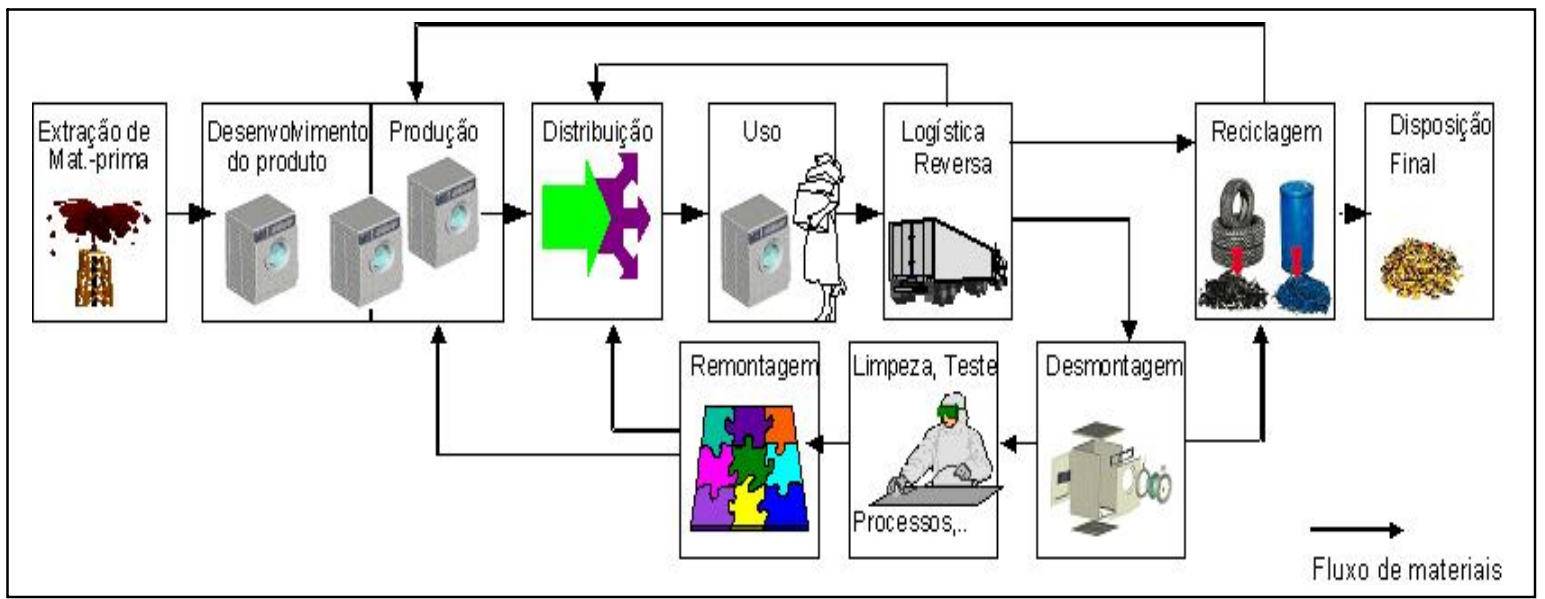

Figura 2: Ciclo de Vida

Fonte: Collaborative Research Center 281, apud AMA, 2004

\subsection{Produção mais Limpa}

Uma metodologia mais simples, que também traz resultados de proteção ambiental definitivos, com vantagens técnicas e econômicas é a Produção mais Limpa, cuja prioridade de sua implantação está baseada na origem da geração de resíduos, buscando soluções nos processos produtivos da empresa. A PmaisL é uma estratégia aplicada na produção e nos produtos, a fim de economizar e maximizar a eficiência do uso de energia, matérias-primas e água e, ainda, minimizar ou reaproveitar resíduos gerados. Ela tem procedimentos simples e econômicos, podendo chegar a um número maior de empresas, pois a análise é feita compreendendo apenas a unidade fabril em questão, sem considerar a cadeia produtiva como um todo, ou seja, fornecedores e clientes não são foco de estudo.

Segundo o Comitê Empresarial Brasileiro de Desenvolvimento Sustentável (CEBDS, 2005), a PmaisL, com seus elementos essenciais, adota uma abordagem preventiva, em resposta à responsabilidade financeira adicional, trazida pelos custos de controle da poluição e dos tratamentos de "fim de tubo", assim como auxilia as empresas a adotarem práticas de fabricação através de um novo conceito de produção e consumo. Com a finalidade de promover o desenvolvimento sustentável nas micro e pequenas empresas do país, foi criada a 
Rede Brasileira de Produção mais Limpa, que difunde o conceito de ecoeficiência e a metodologia de PmaisL, como instrumentos para aumentar a competitividade, a inovação e a responsabilidade ambiental no setor produtivo brasileiro. O programa visa desenvolver uma nova consciência ambiental, evidenciando que a preocupação com as questões ambientais é uma forma inteligente de ganhar dinheiro também.

A PmaisL é uma forma de produzir melhor, gastando menos, e que nem sempre a alteração em um processo depende de investimentos financeiros. Desta forma, a PmaisL propõe que as empresas invistam em tecnologias para redução de resíduos. Para isto, existe uma metodologia que auxilia este processo. De acordo com o Centro Nacional de Tecnologias Limpas (2005), o desafio das empresas é colocar entre seus planos estratégicos a PmaisL, que, comprovadamente, traz benefícios ambientais, econômicos e de saúde ocupacional. Para tanto, é necessária uma mudança de atitude de todos, desde os níveis de diretoria até os níveis operacionais.

A metodologia da PmaisL envolve algumas etapas, e o SEBRAE (2004), apresenta elas da seguinte forma:

a) planejamento e organização - comprometimento da direção e dos funcionários, e formação de equipes de trabalho;

b) pré-avaliação e diagnóstico - estabelecimento de metas para PmaisL e elaboração de fluxogramas, com avaliação de entradas e saídas;

c) avaliação da PmaisL - identificar as ações que podem ser implementadas imediatamente e as que necessitam de análises adicionais mais detalhadas, através de balanços materiais e de energia e informações das fontes e causas da geração de resíduos e emissões;

d) estudo de viabilidade técnica, econômica e ambiental - selecionar as oportunidades viáveis e documentar os resultados esperados;

e) implementação e plano de continuidade - implementar as opções selecionadas e assegurar atividades que mantenham a PmaisL, monitorar e avaliar as oportunidades implementadas, assim como planejar atividades que assegurem a melhoria contínua com a PmaisL. 
A figura 3 demonstra a metodologia de implantação da PmaisL.

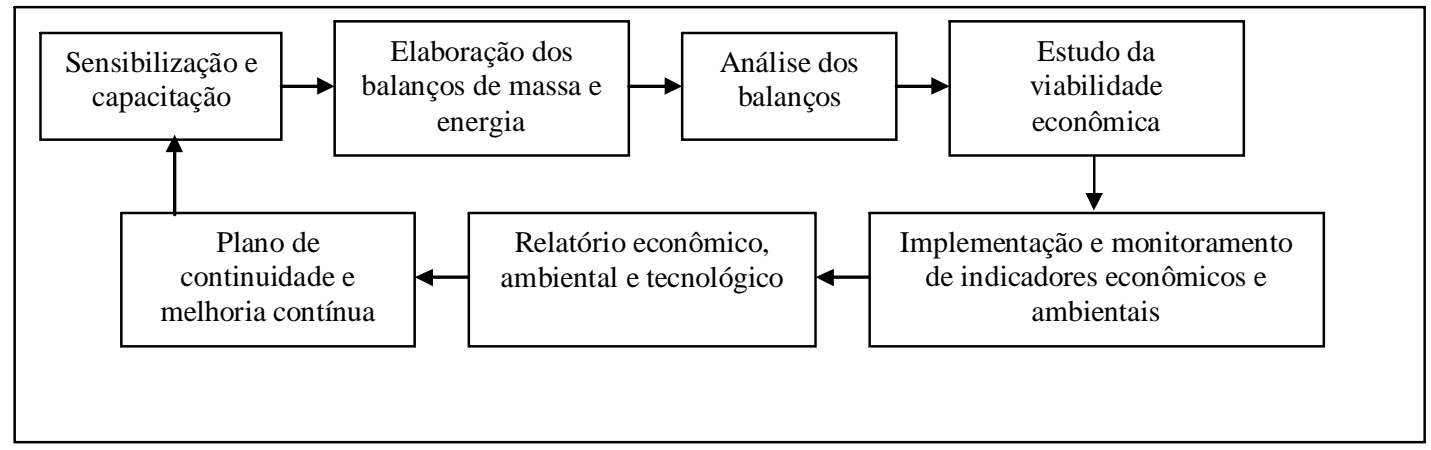

Figura 3: Metodologia de implantação da PmaisL

Fonte: Adaptado de Kraemer, 2002.

Evidencia-se que a PmaisL é uma ferramenta de fácil implementação, pois através da participação de uma equipe coesa e com a cultura disseminada na organização, resultados extremamente positivos podem ser obtidos, entre eles: a minimização dos resíduos gerados, através da redução, reuso, reciclagem e a minimização dos recursos empregados.

Nascimento (2005) apresenta outras vantagens provenientes da PmaisL: aumento da eficiência dos processos, aumento da produtividade, redução dos custos operacionais, melhoria da imagem pública, redução dos riscos ambientais e melhoria do desempenho financeiro.

\section{ANÁLISE COMPARATIVA}

Como apresentado anteriormente, a PmaisL é uma ferramenta de caráter gerencial, econômico, ambiental e de melhoria da qualidade, que requer algumas mudanças na organização, no intuito de tornar a empresa mais competitiva para o mercado.

De acordo com Drolshagen (2005): "Boas práticas de housekeeping, em média, significam redução de $10-15 \%$ no consumo de energia e $15-20 \%$ no consumo de água" (ver Gráfico 1). 


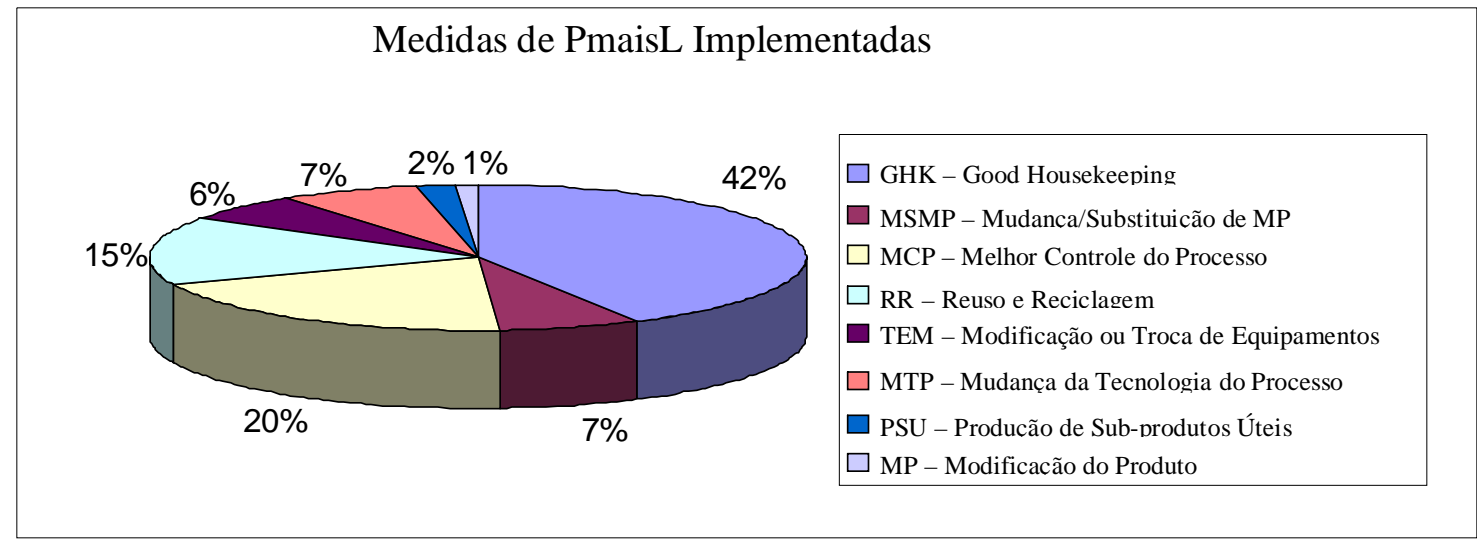

Gráfico 1: Medidas de PmaisL implementadas

Fonte: Drolshagen, 2005.

Dentre as ações implementadas através da PmaisL, no período de 2003 e 2004, o SEBRAE (envolvendo 11 núcleos de Produção mais Limpa), obteve através de investimentos de $\mathrm{R}$ \$ 2.373.737,00 uma redução de custos anuais de $\mathrm{R} \$$ 5.616.268,00 incluindo benefícios ambientais significativos, conforme Tabela 1.

Tabela 1: Benefícios ambientais da PmaisL em 2003 e 2004

\begin{tabular}{|c|c|c|c|}
\hline \multicolumn{4}{|c|}{ Benefícios Ambientais } \\
\hline \multicolumn{2}{|c|}{ Redução do consumo de: } & \multicolumn{2}{|c|}{ Redução na geração de: } \\
\hline Matérias-primas & 167,22 t/ano & Efluentes líquidos & $99.706,90 \mathrm{~m}^{3} /$ ano \\
\hline Água & $11.070 \mathrm{~m}^{3} / \mathrm{ano}$ & Resíduos sólidos & $34.538,39$ t/ano \\
\hline Energia & $350.129,41 \mathrm{KWh} / \mathrm{ano}$ & Resíduos perigosos & 147,12 t/ano \\
\hline Emissões atmosféricas & 3,76 t/ano & Reciclagem externa & 18,20 t/ano \\
\hline Lenha & $1.406 \mathrm{~m}^{3} / \mathrm{ano}$ & Reciclagem interna & $3.296,80$ t/ano \\
\hline Combustíveis & $60,5 \mathrm{~m}^{3} / \mathrm{ano}$ & & \\
\hline Madeira & $716 \mathrm{~m}^{3} /$ ano & & \\
\hline Gás (GLP) & $6.727 \mathrm{Kg} / \mathrm{ano}$ & & \\
\hline
\end{tabular}

Já a ACV tem suas análises direcionadas a parâmetros que envolvem toda a cadeia produtiva do "berço ao túmulo" de um determinado produto, ou seja, são analisados: a extração de matéria-prima, os fluxos de materiais usados, os processos e métodos de fabricação, as formas de transporte empregadas, os tipos de embalagens envolvidas até a disposição final do produto. $\mathrm{Na} \mathrm{ACV}$, a preocupação é com a sustentabilidade de forma ampla, assim as análises realizadas enfocam a categoria do impacto ambiental, associado à 
determinada operação ou produto, do tipo: alteração do habitat natural, aquecimento global, acidificação, ecotoxidade, degradação da camada de ozônio e a escassez dos recursos naturais.

\section{DISCUSSÃO}

Por mais que seja necessário e fácil utilizar programas como a PmaisL, muitos obstáculos ainda estão presentes na sociedade contra a aplicação desse tipo de processo, os quais podem ser citados: barreiras organizacionais, sistêmicas, culturais, técnicas e até mesmo econômicas. Por outro lado, são muitos os benefícios para quem aplica a PmaisL em seu sistema de produção como: benefícios financeiros, comerciais, ambientais e sociais. Com a aplicação da PmaisL, os resíduos serão diminuídos ao máximo, trazendo economia à indústria, assim como a otimização do uso de água e de energia.

Perante o meio ambiente, a PmaisL proporciona uma diminuição significativa quanto aos impactos ambientais, um maior conhecimento dos riscos que a empresa traz à natureza, a redução de resíduos, gases tóxicos e efluentes, além de maiores condições de saúde e segurança aos trabalhadores e à população de forma geral. Pode-se considerar que a PmaisL é uma forma de produzir melhor, gastando menos. Embora algumas alterações no processo precisem de investimento, sempre haverá um retorno econômico embutido. Contudo, existem ações que podem ser implementadas sem a necessidade de recursos financeiros. Logo, trarão mais lucro à organização. Assim, os benefícios ambientais gerados podem reverter-se em um ganho econômico significativo. Este ganho econômico pode estar vinculado não só à redução do uso de água e energia e na redução da geração de resíduos, como também em possíveis multas ambientais que a empresa poderia receber, além da redução nas vendas por uma má publicidade.

Diferentemente da PmaisL, a ACV faz uma análise completa do sistema produtivo, considerando a análise desde o projeto e a realização do produto até a disposição final dos materiais. A ACV tem como objetivo a otimização da utilização dos recursos, e a minimização dos impactos ambientais. Segundo Diogo (2004), "trata-se de um processo ou um conjunto de procedimentos de natureza tecnológica a ser enquadrados num referencial econômico, ambiental e político".

A ACV pode apresentar algumas peculiaridades, que devem ser analisadas e superadas. As etapas de um estudo de ACV, propostas pelas normas ISO, ressaltam a importância da definição clara e objetiva do escopo e dos objetivos do estudo, pois, caso 
contrário, o estudo pode se tornar complexo o suficiente tornando-o inviável. Outro ponto a se destacar é a quantidade de dados necessários e a confiabilidade dos mesmos, pois envolvem toda a cadeia produtiva extrapolando os limites da organização em si e engloba informações oriundas de fornecedores e clientes, que nem sempre disponibilizam estes dados.

Dessa forma, a ACV depende em grande parte dos dados de inventários, os quais devem ser coletados com a maior qualidade possível (KROZER, 1998), e comparar resultados de diferentes estudos de ACV só é possível se as suposições e o contexto de cada estudo forem os mesmos. Outro ponto crítico da ACV é na etapa da avaliação de impacto em que é realizada a classificação, caracterização e valoração dos dados coletados anteriormente. A interpretação dos dados também pode ser outra fonte de dificuldades, pois se faz necessária à análise e interpretação dos resultados obtidos, assim como dos critérios utilizados para o estudo: analisar os resultados, tirar conclusões, explicar as limitações e fornecer recomendações. A interpretação dos resultados deve também evidenciar as limitações que podem tornar os objetivos inicialmente inalcançáveis ou impraticáveis.

As empresas precisam descobrir que não basta pensar apenas em questões econômicas, mas também em questões ambientais e sociais relacionadas a seus produtos, processos e serviços. Ações neste sentido poderão levar a empresa ao sucesso e em alguns casos apenas mantê-la no mercado, onde sua sobrevivência depende de um equilíbrio entre seu desempenho econômico, social e ambiental.

Há de se considerar a ecoeficiência das organizações, por meio do fornecimento de bens e serviços a preços competitivos, que satisfaçam as necessidades humanas e tragam qualidade de vida, ao mesmo tempo em que reduzem progressivamente o impacto ambiental e o consumo de recursos ao longo do ciclo de vida, a um nível, no mínimo, equivalente à capacidade de sustentação estimada da Terra.

\section{CONCLUSÃO}

A ACV encoraja as indústrias a sistematicamente considerar as questões ambientais associadas aos sistemas de produção, pois através dela, a empresa conseguirá trabalhar com objetividade suas questões ambientais através de um ciclo industrial ecológico considerando:

a) a quantidade de recursos naturais renováveis que são utilizados em relação a capacidades destes em se regenerarem; 
b) a utilização de forma racional dos recursos não-renováveis a fim de minimizar ou otimizar esta utilização;

c) o desenvolvimento de novos produtos e processos de forma que gerem menos impactos ambientais;

d) a reutilização ou reciclagem dos mesmos, incluindo uma disposição final segura dos resíduos industriais inevitáveis, assim como do próprio produto após o uso.

Considerando essas idéias, pode-se afirmar que a ACV é uma ferramenta de apoio para a tomada de decisão quanto aos aspectos e impactos ambientais, pois, entre outras aplicações, propõe-se a contribuir para a solução de questões ecológicas, através de uma metodologia bem definida. Enquanto que com a PmaisL a empresa enfoca o aumento da produtividade, através de ações ecologicamente corretas, possibilitando o uso mais racional dos recursos, a PmaisL é uma ferramenta que auxilia as empresas a observarem o quanto é importante ter uma boa imagem perante a sociedade através de ações de preservação ambiental, por meio de processos e produtos, e associar a estes aspectos como a redução de custos e a melhoria do seu desempenho ambiental.

Através do estudo realizado, percebe-se que a PmaisL é uma ferramenta simples de implementar e monitorar, muitas vezes pequenas ações geram grandes resultados. Pode-se considerar que a PmaisL é expressivamente mais simples pois de forma geral sua análise compreende apenas a unidade fabril em questão, o que já não ocorre com a ACV onde dados externos obrigatoriamente precisam ser considerados.

Desta forma a ACV se torna uma ferramenta mais complexa porém mais completa, onde estudos mais aprofundados precisam ser feitos, principalmente no que diz respeito às consequências ao meio-ambiente, assim uma análise de ACV pode levar muito mais tempo do que alternativas de melhorias ambientais (e econômicas) dentro de uma empresa como é a proposta da PmaisL.

É importante ressaltar que as duas ferramentas se preocupam em usar tecnologias para evitar a agressão ao meio ambiente, economizando água e energia, e evitando que resíduos sejam gerados.

A visão tradicional e linear de preservação ambiental, baseada apenas no controle da poluição, através de tecnologias "fim de tubo", utilizadas para o tratamento, minimização e inertização de resíduos, atuando de forma a remediar os efeitos da produção, onde os resíduos gerados são posteriormente tratados, não garante a ecoeficiência e a sustentabilidade ambiental, sendo esta uma tratativa ecologicamente ineficiente. Já a PmaisL e ACV, 
englobam a gestão do sistema de produção, preocupadas com o meio-ambiente, e vieram justamente para responder a questão: De que forma as empresas podem trabalhar de forma a contribuir para o desenvolvimento sustentável do planeta?

Assim, pelo atual crescimento do mercado, os cuidados com o meio ambiente deixam de ser apenas uma fonte de despesas para tornarem-se uma fonte geradora de lucros. Sob a ótica da gestão ambiental, aquilo que antes era um tema sem importância para as empresas água, ar, energia, resíduos em geral e sucatas - transformou-se em uma oportunidade para reduzir, reutilizar e reciclar, agregando valores sustentáveis para as empresas e para a sociedade.

Outros fatores que influenciam as empresas para adoção de posturas ambientalmente responsáveis através da $\mathrm{ACV}$ ou da PmaisL são: percepção das vantagens em termos competitivos; melhoria na imagem perante a sociedade; redução de custos dos seus processos produtivos; adoção de programas de gestão específicos, gerando assim uma ação com resultados ecoeficientes.

O principal objetivo deste estudo foi demonstrar que o crescimento dos negócios está de fato atrelado aos aspectos e impactos ambientais, onde existem questões ambientais voltadas à legislação - que precisam ser obrigatoriamente cumpridas, outras, por uma situação estratégica - representando para empresa um diferencial competitivo, mas, também, há aquela voltada para a sustentabilidade - esta por sua vez precisa ser cada vez mais trabalhada.

Há de se reconhecer ainda que o Desenvolvimento Sustentável só será de fato alcançado se três pontos chaves forem atendidos: o crescimento econômico, o equilíbrio ecológico e o progresso social.

\section{REFERENCIAS}

AMA - Grupo de Adequação Ambiental em Manufatura. V Workshop Internacional de Adequação Ambiental em Manufatura: Engenharia do Ciclo de Vida. 25 de novembro de 2004, São Carlos, SP. Participação do coordenador do Collaborative Research Center 281, Prof. Dr.-Ing. Günther Seliger e do vice-coordenador Dipl.-Ing. Carsten Franke, ambos da Universidade Técnica de Berlin. Disponível em: <http://www.numa.org.br/Vworkshop\%20ama.htm>. Acesso em: 28 de junho de 2005.

CEBDS - Centro Empresarial Brasileiro de Desenvolvimento Sustentável. Produção mais Limpa - Conceitos. 2005. Disponível em: < http://www.cebds.org.br/cebds/eco-pmaislconceito.asp>. Acesso em: 15 de agosto de 2005. 
Centro Nacional de Tecnologias Limpas. A produção mais limpa na micro e pequena empresa. CEBDS - Conselho Empresarial Brasileiro para o Desenvolvimento Sustentável. 2005 - Guia de Produção mais Limpa - Senai - RS Disponível em: <http://www.pmaisl.com.br/publicacoes/guiadepmaisl/guia-da-pmaisl.pdf $>$. Acesso em: 23 de dezembro de 2005.

CHEHEBE, José Ribamar. Análise do Ciclo de Vida dos Produtos: Ferramenta gerencial da ISO 14000. Rio de Janeiro: Qualitymark, 1998.

DIOGO, A. Correia. MARGARIDO, Fernanda. BORDADO, J. C. O sistema produtivo: Tecnologias relevantes no metabolismo da economia. Universidade Técnica de Lisboa.2004. Disponível em: 〈http://seminarios.ist.utl.pt/04-05/des/html/sessoes/sessao6.shtml〉. Acesso em: 01 de junho de 2005.

DROLShaGEN, Márcia. PmaisL nas PMEs: Oportunidades e inovação. Congresso Ibero Americano sobre Desenvolvimento Sustentável. 2005. Disponível em: $<$ http://cebds.dynalias.net/cebds/docnoticia/sust2005-Marcia-Drolshagen.pdf $>$. Acesso em: 15 de agosto de 2005.

DUARTE, Marcos Daniel. Caracterização da rotulagem ambiental de produtos. Dissertação de Mestrado, UFSC, Florianópolis, SC, 1997.

KROZER, J.; VID, J.C. How to get LCA in the right direction? Journal of cleaner production, v. 6, 1998, p. 53-61.

KRAEMER, Tânia Henke. Modelo econômico de controle e avaliação de impactos ambientais. Tese de Doutorado em Eng. de Produção, UFSC, 2002.

NASCIMENTO, Luis Felipe, Produção mais limpa. Programa de Pós-Graduação em Administração de Empresas. UFRGS - Escola de Administração, 2005.

Disponível em: <http://disciplinas.adm.ufrgs.br/engambiental/> . Acesso em: 13 de junho de 2005.

NBR ISO 14040 - Gestão Ambiental: Avaliação do Ciclo de Vida - Princípios de estrutura. ABNT. Rio de Janeiro. 2001.

SEBRAE. Relatório de Atividades dos Núcleos Regionais de Produção mais Limpa CEBDS Conselho Empresarial Brasileiro para o Desenvolvimento Sustentável. SEBRAE 2003/2004. Rio de Janeiro, outubro/2004. 